\title{
Superconductivity studies on $\mathrm{Tl}-\mathrm{Th}-\mathrm{Sr}-\mathrm{Ca}-\mathrm{Cu}-\mathrm{O}$ system
}

\author{
K A THOMAS, U V VARADARAJU, G V SUBBA RAO, C V \\ TOMY* and S K MALIK* \\ Materials Science Research Centre, Indian Institute of Technology, Madras 600036, India \\ *Solid State Physics Group, Tata Institute of Fundamental Research, Bombay 400005, India
}

\begin{abstract}
Superconductivity with a maximum $T_{c . z e r o}$ of $58 \mathrm{~K}$ for $x>0.75$ has been observed in a nominal starting composition. $\mathrm{Th}_{x} \mathrm{Tl}_{2} \mathrm{Sr}_{2} \mathrm{Ca}_{2} \mathrm{Cu}_{3} \mathrm{O}_{v}$. X-ray data show that the 1212 phase gets stabilized due to part substitution of Th at the Tl-site for $x \geqslant 0 \cdot 25$; however, $T_{c}$ is low for $x$ $<0.75$.
\end{abstract}

Keywords. Thallium; thorium: strontium; calcium: copper; oxides: superconductors.

\section{Introduction}

High $T_{c}$ superconductivity in single and double layer thallium cuprates having the general formula $\mathrm{Tl}_{m} \mathrm{Ba}_{2} \mathrm{Ca}_{n-1} \mathrm{Cu}_{n} \mathrm{O}_{m+2(n+1)} ; m=1,2 ; n=1-3$ is now well known (Sheng et al 1988a, b; Parkin et al 1988a, b; Rao and Raveau 1989; Ramakrishnan and Rao 1989). The compound with $m=2$ and $n=3$ viz. $\mathrm{Tl}_{2} \mathrm{Ba}_{2} \mathrm{Ca}_{2} \mathrm{Cu}_{3} \mathrm{O}_{10}$ (2223) possesses the highest known $T_{\text {c,zero }}$ of $125 \mathrm{~K}$. These phases possess a layered structure with distorted rock salt-type layers of $\mathrm{TlO}$ and oxygen-deficient perovskite-type layers $\left(\mathrm{ACuO}_{3-y}\right)$ with $\mathrm{A}=(\mathrm{Ba}, \mathrm{Ca})$. It is now well established that the Ca-site of the 1212 phase $\left(\mathrm{TlBa}_{2} \mathrm{CaCu}_{2} \mathrm{O}_{7} ; T_{c}=80 \mathrm{~K}\right)$ can be substituted either partly or fully by rare earths or Y to give isostructural phases (Manako et al 1988; Ganguli et al 1989; Rao et al 1989; Martin et al 1989). The fully substituted phases (e.g. $\mathrm{TlBa}_{2}\left(\mathrm{Ln} / \mathrm{Y} \mathrm{Cu}_{2} \mathrm{O}_{7}\right)$ are not superconducting but semiconducting (Manako et al 1988; Ganguli et al 1989; Rao et al 1989; Martin et al 1989).

It is difficult to realize a 1212 phase in pure form in the $\mathrm{Tl}-\mathrm{Sr}-\mathrm{Ca}-\mathrm{Cu}-\mathrm{O}$ system. However it is possible to stabilize such a 1212 phase (with $\mathrm{Sr}$ ) by partially substituting either (i) the Tl-site by Pb, Bi or Th (Subramanian et al 1989; Li and Greenblatt 1989; Thomas et al 1990a) or the Ca-site by Y or rare earth (Ganguli et al 1989; Rao et al 1989; Liang et al 1989; Sheng et al 1989; Thomas et al 1990a). Recently Rao et al (1989) have shown that the 1212 phase can also be stabilized without a calcium site occupancy, viz. phase of the type. $\mathrm{Tl}_{1} \mathrm{Sr}_{3-x} \mathrm{Ln}_{x} \mathrm{Cu}_{2} \mathrm{O}_{1}$. All the above phases are metallic and exhibit superconductivity. However, the $T_{\text {c.zcro }}$ values do depend on the metal ion substituting at the T1- or Ca-site (Ganguli et al 1989; Rao et al 1989; Subramanian et al 1989; Li and Greenblatt 1989; Liang et al 1989; Sheng et al 1989).

In our earlier studies (Thomas et al 1990a) we have shown that when $\mathrm{Sm}$ is substituted at the $\mathrm{Ca}$-site in the $\mathrm{Tl}-\mathrm{Sr}-\mathrm{Ca}-\mathrm{Cu}-\mathrm{O}$ system a nominal composition of $x$ $=0.25$ is necessary and sufficient to stabilize the 1212 structure in a nominal composition $\mathrm{Sm}_{x} \mathrm{Tl}_{2} \mathrm{Sr}_{2} \mathrm{Ca}_{2} \mathrm{Cu}_{3} \mathrm{O}_{y}$ and induce a $T_{\text {c.zero }}$ of $68 \mathrm{~K}$. We thought it interesting to examine whether a similar behaviour would be exhibited by the nominal $\mathrm{Th}_{x} \mathrm{Tl}_{2} \mathrm{Sr}_{2} \mathrm{Ca}_{2} \mathrm{Cu}_{3} \mathrm{O}_{y}$ system. The present studies show that indeed the 1212 superconducting phase gets stabilized with $T_{\text {c.zero }}$ of $58( \pm 1 \mathrm{~K})$ for $x=0.75$ and 1.00 but the $T_{c, \text { zero }}$ are lower for $x=0.50$ and 0.25 . 


\section{Experimental}

Compounds with nominal composition of $\mathrm{Th}_{x} \mathrm{Tl}_{2} \mathrm{Sr}_{2} \mathrm{Ca}_{2} \mathrm{Cu}_{3} \mathrm{O}_{y}(x=0.0$ to 1.0$)$ have been synthesized using a matrix method starting from high purity $\mathrm{Tl}_{2} \mathrm{O}_{3}$ (Cerac, UK; $99.9 \%$ ), $\mathrm{ThO}_{2}$ (Fluka, 99.9\%), $\mathrm{SrCO}_{3}\left(\mathrm{Cerac}, 99.9 \%\right.$ ), $\mathrm{CaCO}_{3}(\mathrm{BDH}, 99 \%$ ) and $\mathrm{CuO}$ $\left(99.99 \%\right.$ ). Initially mixtures of $\mathrm{SrCO}_{3}, \mathrm{CaCO}_{3}$ and $\mathrm{CuO}$ were thoroughly mixed in the ratio $2: 2: 3$ and heated at $950^{\circ} \mathrm{C}$ in air for $24 \mathrm{~h}$ with several intermittent grindings and heatings. The resulting multiphase mixture, $\mathrm{Sr}_{2} \mathrm{Ca}_{2} \mathrm{Cu}_{3} \mathrm{O}_{y}$ served as the master composition. Appropriate amounts of $\mathrm{Tl}_{2} \mathrm{O}_{3}$ and $\mathrm{ThO}_{2}$ were then added to the master composition to give a nominal composition of $\mathrm{Th}_{x} \mathrm{Tl}_{2} \mathrm{Sr}_{2} \mathrm{Ca}_{2} \mathrm{Cu}_{3} \mathrm{O}_{y}$, thoroughly ground and pelletized. The pellets were then introduced into a preheated furnace $\left(900^{\circ} \mathrm{C}\right)$ in flowing oxygen gas and kept for $5 \mathrm{~min}$. The pellets were then taken out of the hot zone and allowed to cool to room temperature by furnace shut-off in the flowing $\mathrm{O}_{2}$-gas. The samples were characterized by X-ray powder diffraction (XRD, Philips PW 1140, Ni-filter). The theoretical $d$ values and intensities were obtained using the LAZYPULVERIX programme (Siemens Computer, model 7580E). The lattice parameters were calculated from the high angle $(h k l)$ reflections by the least squares (LSQ) method. Four-probe d.c. electrical resistivity was measured in the range 300$15 \mathrm{~K}$ on all the samples using a closed cycle He-refrigerator (CTI-Cryogenics, USA, model 21C) using ultrasonically-soldered In-metal contacts. AC magnetic susceptibility experiments were carried out down to $15 \mathrm{~K}$ using a closed cycle He-refrigerator (Sumitomo, Japan, model SCR 204 T).

\section{Results and discussion}

The phases were black in colour and stable under normal atmospheric conditions. The XRD patterns of all the compositions (except when $x=0 \cdot 0$, viz. $\mathrm{Tl}_{2} \mathrm{Sr}_{2} \mathrm{Ca}_{2} \mathrm{Cu}_{3} \mathrm{O}_{y}$ ) indicated the formation of 1212 as the major phase with other impurity phases (figure 1). The LSQ-fitted tetragonal lattice parameters are given in table 1 . We notice a decrease in the $c$ lattice parameter with increasing $x$ whereas $a$ lattice parameter remains almost the same in the range $0.25 \leqslant x \leqslant 0.75$. The phases with $x \geqslant 0.50$ showed fewer impurity lines compared to that with $x \leqslant 0 \cdot 25$. The theoretically calculated and observed intensities of the $(h k l)$ lines agree only when we assume fractional occupancy of the Th at the Tl-site.

All the phases presently studied are metallic and exhibit superconductivity. The resistivity vs temperature plots are shown in figure 2 and the $T_{c}$ data are summarized in table 1. $T_{c, \text { zero }}$ values of $58 \mathrm{~K}$ and $57 \mathrm{~K}$ were noticed when $x=1.00$ and 0.75 respectively. The $T_{c \text {, zero }}$ are lower $(<50 \mathrm{~K})$ when $x=0.25$ and 0.50 . The parent compound, viz. $\mathrm{Tl}_{2} \mathrm{Sr}_{2} \mathrm{Ca}_{2} \mathrm{Cu}_{3} \mathrm{O}_{y}$ (nominal composition) showed a $T_{c, \text { zero }}$ of $22 \mathrm{~K}$ with a step $(50 \%$ drop in resistivity) around $80 \mathrm{~K}$ (not shown in figure 2 ). For the same phase superconductivity with a $T_{c, \text { zero }}$ ranging from $20-80 \mathrm{~K}$, depending on the details of the preparative conditions, has been reported in the literature (Sheng et al 1988a; Hayri and Greenblatt 1988).

Magnetic susceptibility vs temperature plots for the system $\mathrm{Th}_{x} \mathrm{Tl}_{2} \mathrm{Sr}_{2} \mathrm{Ca}_{2} \mathrm{Cu}_{3} \mathrm{O}_{y}$ $(x=0 \cdot 25,0 \cdot 50,0 \cdot 75)$ are shown in figure 3 . All the samples showed transition to diamagnetic state indicating that the superconducting properties of these phases are bulk in nature. For $x=0.25$ and 0.50 , the $T_{c}$ (onset of diamagnetism) measured from $\chi-T$ 


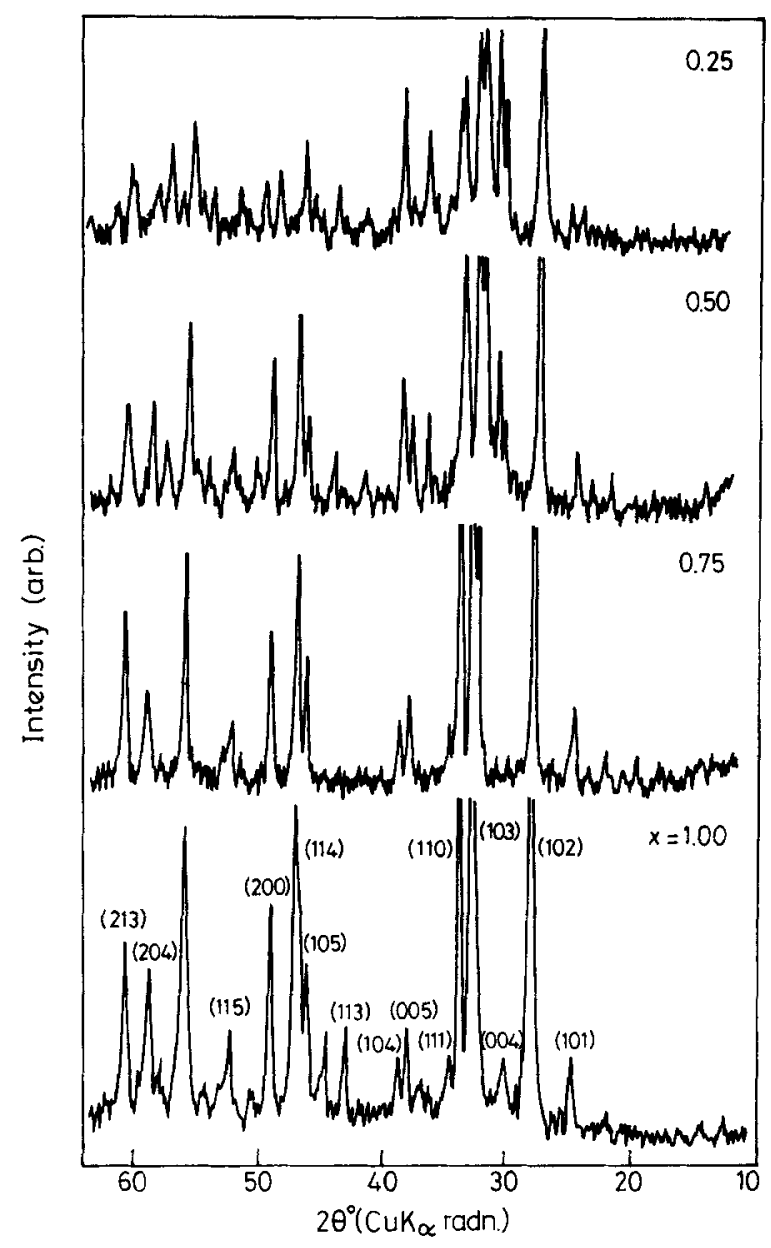

Figure 1. XRD patterns of the phases with the nominal composition, $\mathrm{Th}_{x} \mathrm{Tl}_{2} \mathrm{Sr}_{2} \mathrm{Ca}_{2} \mathrm{Cu}_{3} \mathrm{O}_{y} x$ values are indicated. The phases can be indexed as the tetragonal 1212 structure with $T h$ partly occupying the Tl-site.

Table 1. Structure and $T_{v}$ data on $\mathrm{Th}_{x} \mathrm{Tl}_{2} \mathrm{Sr}_{2} \mathrm{Ca}_{2} \mathrm{Cu}_{3} \mathrm{O}_{y}$.

\begin{tabular}{|c|c|c|c|c|c|c|}
\hline \multirow{2}{*}{$\begin{array}{c}\text { Composition } \\
x\end{array}$} & \multicolumn{2}{|c|}{$\begin{array}{c}\text { Tetragonal } \\
\text { lattice param, } \AA\end{array}$} & \multirow{2}{*}{$\begin{array}{c}T_{c} \\
\text { (onset) } \\
K\end{array}$} & \multirow{2}{*}{$\begin{array}{c}T_{c} \\
\text { (zero) } \\
K\end{array}$} & \multirow{2}{*}{$\begin{array}{l}\rho_{300 \mathrm{~K}} \\
\mathrm{~m} \Omega \mathrm{cm}\end{array}$} & \multirow{2}{*}{$\begin{array}{c}\left(1 / \rho_{300 \mathrm{~K}}\right)(\partial \rho / \partial T) \times 10^{-3} \\
(100-300 \mathrm{~K})\end{array}$} \\
\hline & $a$ & $c$ & & & & \\
\hline 0.0 & - & - & 40 & 22 & $1 \cdot 8$ & $2 \cdot 4$ \\
\hline 0.25 & 3.81 & $12 \cdot 08$ & 70 & 46 & 7.0 & 1.4 \\
\hline 0.50 & 3.80 & $12 \cdot 07$ & 70 & 52 & $8 \cdot 8$ & 1.8 \\
\hline 0.75 & 3.81 & $12 \cdot 03$ & 80 & 57 & $9 \cdot 1$ & 20 \\
\hline 1.00 & $3 \cdot 77$ & 11.94 & 85 & 58 & $10 \cdot 6$ & $2 \cdot 0$ \\
\hline
\end{tabular}




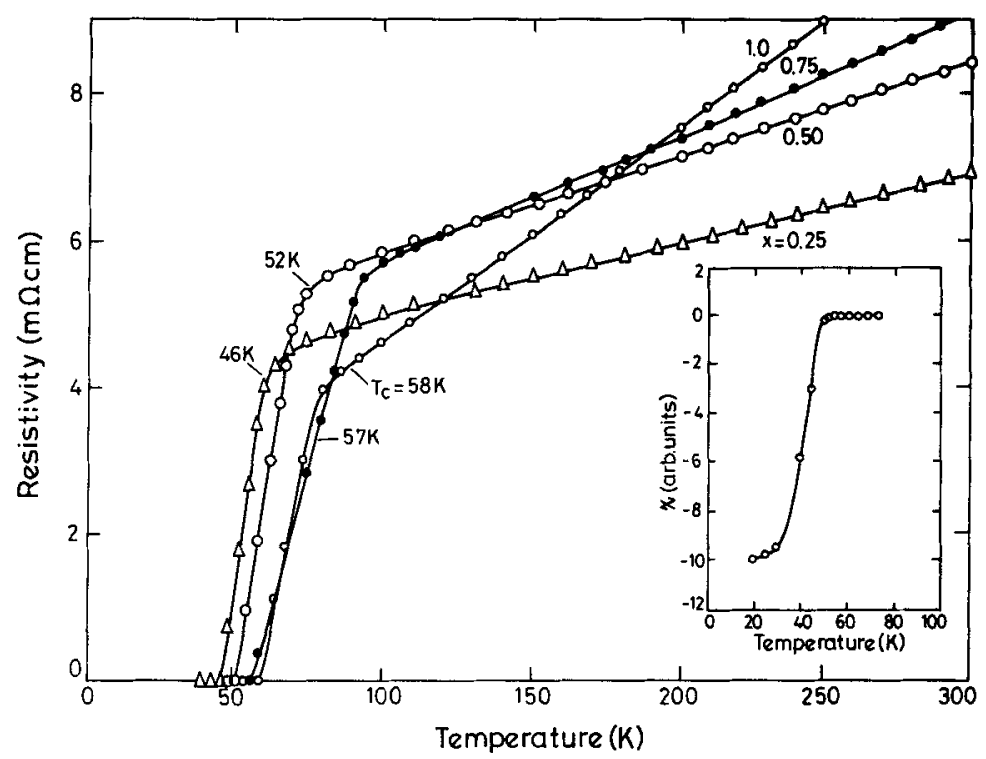

Figure 2. $\rho$-T plots for $\mathrm{Th}_{x} \mathrm{Tl}_{2} \mathrm{Sr}_{2} \mathrm{Ca}_{2} \mathrm{Cu}_{3} \mathrm{O}_{y}$ showing superconducting transitions. The $x$ values are indicated. Inset shows $\chi-T$ plot for $x=1 \cdot 0$.

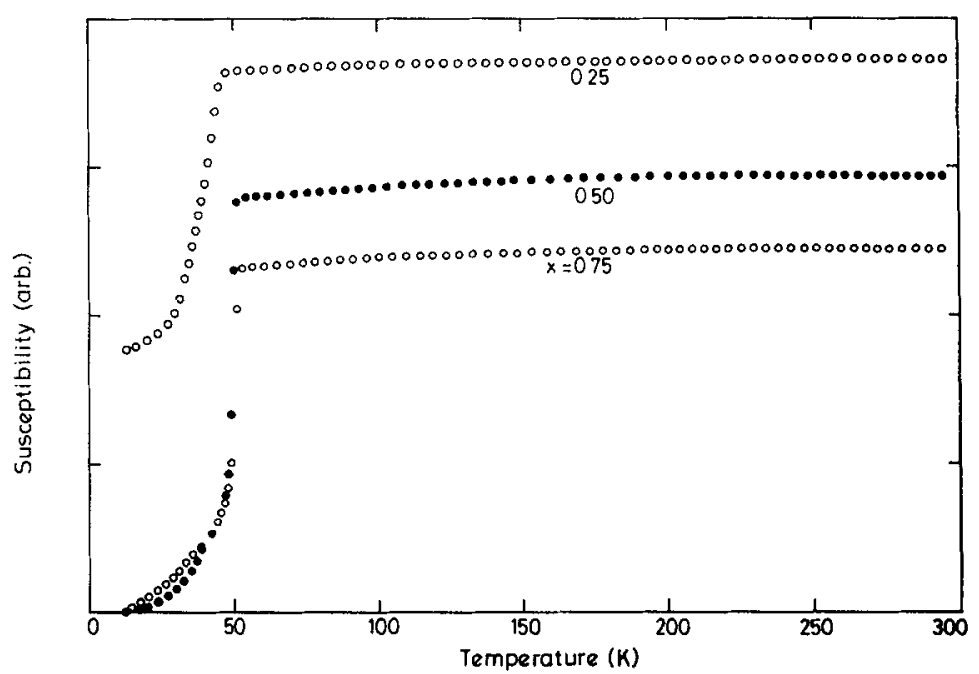

Figure 3. $\chi$ - $T$ plots for the nominal $\mathrm{Th}_{x} \mathrm{Tl}_{2} \mathrm{Sr}_{2} \mathrm{Ca}_{2} \mathrm{Cu}_{3} \mathrm{O}_{y}$ compositions showing onset of diamagnetism (superconductivity). The $x$ values are indicated.

measurements ( $48 \mathrm{~K}$ and $50 \mathrm{~K}$ respectively) is only slightly different from the $T_{\text {c,zero }}$ encountered in $\rho$ - $T$ measurements (table 1, figure 2 ). For $x=0.75$ and 1.0 , the $T_{\text {c.zero }}$ of $57 \mathrm{~K}$ and $58 \mathrm{~K}$ respectively are larger than the $T_{c}$ obtained from $\chi-T$ measurements $(50 \mathrm{~K})$.

We notice a larger room temperature resistivity $\left(\rho_{300 \mathrm{~K}}\right)$ for the Th-containing phases compared to that for the nominal $\mathrm{Tl}_{2} \mathrm{Sr}_{2} \mathrm{Ca}_{2} \mathrm{Cu}_{3} \mathrm{O}_{y}$ phase (table 1 and figure 2). This suggests that addition of $\mathrm{Th}$, which will partly substitute the Tl-site, stabilizes a 
1212 phase and drives the parent phase towards the metal-semiconductor phase boundary and induce superconductivity around $50 \mathrm{~K}$. Similar behaviour was observed in the $\mathrm{Ln}_{x} \mathrm{Tl}_{2} \mathrm{Sr}_{2} \mathrm{Ca}_{2} \mathrm{Cu}_{3} \mathrm{O}_{y}$ (Thomas et al 1990a) and $\mathrm{M}_{x} \mathrm{Tl}_{2} \mathrm{Ba}_{2} \mathrm{Cu}_{3} \mathrm{O}_{y}$ (Thomas et al 1990b) systems.

\section{Conclusions}

The present study indicates that addition of Th stabilizes the 1212 structure in the $\mathrm{Tl}-$ $\mathrm{Sr}-\mathrm{Ca}-\mathrm{Cu}-\mathrm{O}$ system and induces superconductivity with a $T_{c, \text { zero }}$ of $58 \mathrm{~K}$. It appears that the added $\mathrm{Th}$ occupies the $\mathrm{Tl}$-site and not the $\mathrm{Ca}$-site in the 1212 lattice as it occurs in the $\mathrm{Ln}-\mathrm{Tl}-\mathrm{Sr}-\mathrm{Ca}-\mathrm{Cu}-\mathrm{O}$ system. While a nominal composition of $x=0.25$ in $\mathrm{Th}_{x} \mathrm{Tl}_{2} \mathrm{Sr}_{2} \mathrm{Ca}_{2} \mathrm{Cu}_{3} \mathrm{O}_{y}$ is sufficient to stabilize the 1212 phase, a $T_{c \text {,zero }}$ of $57 \mathrm{~K}$ is induced only for $0.75 \leqslant x \leqslant 1.0$.

\section{Acknowledgement}

Thanks are due to PMB, DST, Govt. of India for the award of research grants.

\section{References}

Ganguli A K, Nagarajan R, Nanjundaswamy K S and Rao C N R 1989 Mater. Res. Bull. 24103

Hayri E A and Greenblatt M 1988 Physica C156 775

Li S and Greenblatt M 1989 Physica C157 365

Liang J K, Zhang Y L, Rao G H, Cheng X R, Xie S S and Zhao Z X 1989 Solid State Commun. 70661

Manako T, Shimakawa Y, Kubo Y, Satoh T and Igarashi H 1988 Physica C156 315

Martin C, Bourgault D, Michel C, Hervieu H and Raveau B 1989 Mod. Phys. Lett. B3 93

Parkin S S P, Lee V Y, Engler E M, Nazzal A I, Huang T C, Gorman G, Savoy R and Beyers R 1988a Phys. Rev. Lett. 602539

Parkin S S P, Lee V Y, Nazzal A I, Savoy R, Huang T C, Gorman G and Beyers R 1988b Phys. Rev. B38 6531 Ramakrishnan T V and Rao C N R 1989 J. Phys. Chem. 934414

Rao C N R, Ganguli A K and Vijayaraghavan R 1989 Phys. Rev. B40 2565

Rao C N R and Raveau B 1989 Acc. Chem. Res. 22106

Sheng Z Z, Hermann A M, Vier D C, Schultz S, Oseroff S B, George D J and Hazen R M 1988a Phys. Rev. B38 7074

Sheng Z Z and Hermann A M 1988b Nature (London) 332 55, 138

Sheng Z Z, Sheng L, Fei X and Hermann A M 1989 Phys. Rev. B39 2918

Subramanian M A, Torardi C C, Gopalakrishnan J, Gai P L, Calabrese J C, Askew T R, Flippen R B and Sleight A W 1989 Science 242249

Thomas K A, Varadaraju U V, Subba Rao G V, Tomy C V and Malik S K 1990a Mater. Res. Bull. 25465

Thomas K A, Varadaraju U V, Subba Rao G V. Tomy C V and Malik S K $1990 \mathrm{~b}$ J. Solid State Chem. 88177 